(2) Open Access Full Text Article

CLINICALTRIAL REPORT

\title{
Antiaging efficacy of melatonin-based day and night creams: a randomized, split-face, assessor-blinded proof-of-concept trial
}

This article was published in the following Dove Press journal:

Clinical, Cosmetic and Investigational Dermatology

\author{
Massimo Milani' \\ Adele Sparavigna $^{2}$ \\ 'Medical Department, Cantabria Labs \\ Difa Cooper, Caronno Pertusella, \\ ${ }^{2}$ Derming, Clinical Research and \\ Bioengineering Institute, Milan, Italy
}

Background: Skin is a complete and independent melatoninergic system. At the skin level, melatonin (Mel) acts as a relevant antioxidant and cytoprotective substance. Topical application of $\mathrm{Mel}$ is considered meaningful, since it can easily penetrate the stratum corneum. Exogenous Mel can be expected to represent a potent antioxidative defense system against skin aging mechanisms. Day and night creams containing Mel, carried in lipospheres (Melatosphere ${ }^{\mathrm{TM}}$ ), have been developed (Nutriage SPF 30 day cream and Nutriage night cream).

Study aim: The aim of this study was to evaluate the efficacy of a Mel-based cream as antiaging treatment.

Subjects and methods: In a randomized, split-face, assessor-blinded, prospective 3-month study, 22 women (mean age 55 years) with moderate-severe skin aging were enrolled (clinical trial registration number: NCT03276897). Study products were applied in the morning (Nutriage day cream) and evening (Nutriage night cream) on the right or left side of the face. Primary outcomes were: 1) clinical evaluation of wrinkles' grade (crow's feet and nasolabial folds), surface microrelief, skin tonicity (resistance to pinching and traction, recovery after pinching) and skin dryness and 2) instrumental evaluation of skin roughness and 3D photographic documentation (Vectra H1 images system). Assessments of both clinical and instrumental evaluations were performed at baseline and after 1, 2 and 3 months of treatment by an investigator unaware of treatment allocation. Results: All the subjects completed the study. Crow's feet was reduced significantly ( $p=0.05$ ) by $-15 \%$ with the creams in comparison with the non-treated side after 3 months. At the end of the study, surface microrelief $(-26.5 \%)$, skin profilometry $(-13 \%)$, skin tonicity $(+30 \%)$ and skin dryness $(-59.5 \%)$ significantly improved with active treatment. Both products were well tolerated.

Conclusion: In women with skin aging, Mel-based creams improved significantly skin tonicity and skin hydration with a significant reduction in skin roughness, supporting the skin antiaging effect of this molecule applied topically.

Keywords: melatonin, skin aging, assessor-blinded trial

\section{Introduction}

Skin is a complete and independent melatoninergic system. ${ }^{1,2}$ Skin can synthesize melatonin (Mel), and skin cells express melatonin receptors MT1 and MT2. ${ }^{3,4} \mathrm{Mel}$ is a pleiotropic molecule. ${ }^{5}$ At the skin level, Mel acts as a relevant antioxidant and cytoprotective substance and plays a fundamental role in maintaining the homeostasis of the skin. ${ }^{6}$ Skin Mel, and most of its metabolites, exerts a strong protection against oxidative stress and ultraviolet radiation. ${ }^{2,7-9}$ Cutaneous melatoninergic and metabolic systems can act as auto/paracrine protectors against environmentally induced damage. ${ }^{10}$ Skin
Correspondence: Massimo Milani Medical Department, Cantabria Labs, Difa Cooper, Via Milano 160, Caronno Pertusella, VA, Italy

Email massimo.milani@difacooper.com 
rapidly metabolizes Mel. ${ }^{11}$ Therefore, exogenous Mel can be expected to represent one of the most potent antioxidative defense systems against skin aging mechanisms. ${ }^{12}$ Oral administration of Mel shows a prominent first-pass effect with very low systemic level. ${ }^{13,14}$ Topical administration could circumvent this problem. Topical application of Mel is considered meaningful, since it can penetrate the stratum corneum due to its distinct lipophilic chemical structure. ${ }^{15}$ So far, no clinical data regarding the efficacy of topical Mel as an antiaging molecule has been reported. Recently, day and night creams containing Mel, vehiculated in lipospheres (Melatosphere ${ }^{\mathrm{TM}}$ ), have been developed (Nutriage day cream and Nutriage night cream; Cantabria Labs, Difa Cooper, Caronno Pertusella, VA, Italy). The liposphere carrier system could further improve the penetration of the Mel molecules through the skin layers. ${ }^{16}$

\section{Study aim}

The aim of this study was to assess the antiaging effect of $0.1 \%$ Mel-based creams (a day formulation and a night formulation).

\section{Subjects and methods}

We planned a randomized, split-face, assessor-blinded, prospective 3-month study.

\section{Subjects}

In all, 22 women (mean age 55 years) with moderate-severe skin aging were enrolled after receiving their written informed consent. The subjects also provided consent to the publication of any pictures. The inclusion criterion was women aged $\geq 45$ years with moderate-severe facial skin aging (Glogau score of 3 or 4). The exclusion criteria were any acute or chronic skin conditions, which could interfere with the parameters of evaluation and a positive history of allergic contact dermatitis to any of the component of the creams. Study products were applied one in the morning (Nutriage day cream) and one in the evening (Nutriage night cream) on the right or left side of the face according to a randomization list. The randomization list was generated using a dedicated software. The local institutional review board (IRB; Independent Ethical Committee, c/o Derming Clinic, Milan, Italy) approved the trial protocol on March 10, 2017 (clinical trial registration Number: NCT03276897). The study was conducted in accordance with the ethical principles of the Declaration of Helsinki and consistent with the Good Clinical Practice (GCP) regulatory requirements. ${ }^{17}$ Compliance to the treatment was evaluated counting the returned used cream blisters at visits during months 1,2 and 3 .

\section{Study outcomes}

Primary outcomes were: 1) clinical evaluation of wrinkles' grade (crow's feet and nasolabial folds), surface microrelief, skin firmness/tonicity (resistance to pinching and traction, recovery after pinching) and skin dryness and 2) instrumental evaluation of skin roughness and 3D photographic documentation (Vectra H1 images system). Assessments of both clinical and instrumental evaluations were performed at baseline and after 1, 2 and 3 months of treatment by an investigator unaware of treatment allocation. Secondary outcome was the local tolerability of the products evaluated. Sky dryness was evaluated using a 6-point score scale (from 0: very hydrated to 5: very dry). Resistance to traction, resistance to pinching and recovery after pinching were evaluated using a 5-point score scale (from 0: very strong to 4: very weak). Wrinkles' grade and surface microrelief were scored visually on the base of high-definition pictures using the Glogau ${ }^{18}$ (crow's feet) and Monheit et $\mathrm{al}^{19}$ (nasolabial) photographic 6-point scales. Surface microrelief was evaluated using a 4-point scale (from 1: very regular to 4: very irregular). Instrumental evaluation of wrinkles using profilometry was performed at the crow's feet area using Primos compact portable device (GFMesstechinck, Konstanz; Germany). The Primos software is able to elaborate 3D representations of skin wrinkles and to measure skin principal profilometric parameters. Pictures with the Vectra device were performed in a standardized (distance and illumination) manner with the subjects with open eyes and relaxed face muscles. Finally, the Spiderming ${ }^{\mathrm{TM}}$ radial graph was used to "visualize" the global antiaging effect of the treatment. ${ }^{20}$ This graph (Derming S.r.l. Clinical Research Institute, Milan, Italy) allows the results to be evaluated and the effect of an antiaging cosmetic product to be visualized, taking into account that a smaller area of the graph coincides with a younger skin; the activity of the products in terms of antiaging activity could be quantified.

\section{Statistical analysis and sample size calculation}

Statistical analysis was performed using GraphPad Statistical Software (GraphPad Software, Inc., La Jolla, CA, USA). Continuous variables were expressed as mean \pm standard deviation (SD). The primary outcomes of the study were to evaluate scores of skin parameters at month 3 in comparison with baseline values (intragroup evaluation) using paired nonparametric test (Wilcoxon test) and to compare at month 3 , the same skin parameters between treated and non-treated sides (intergroup evaluation) using unpaired nonparametric test (Mann-Whitney test). The secondary outcome was to 
evaluate the evolution of the skin variables during the study at each visit (baseline, month 1, month 2 and month 3 ) using analysis of variance (ANOVA) test for repeated measures. Inferential statistical analysis was done based on intentionto-treat principle. In view of the proof-of-concept nature of the present trial, a formal sample size calculation was not performed. We decided to enroll at least 20 evaluable subjects.

\section{Results}

The trial was conducted between March 2017 and September 2017 in an outpatient derma-esthetic clinic. All the subjects completed the study. In comparison with baseline value, skin dryness was significantly ( $p=0.001$; Wilcoxon test) reduced by $59.5 \%$ (from $3.6 \pm 0.7$ to $1.5 \pm 0.4$ ) on the side treated with Mel creams. Skin dryness score was significantly lower in the active-treated side in comparison with the non-treated side ( $p=0.01$; Mann-Whitney $U$ test; $1.5 \pm 0.4$ vs $3.5 \pm 0.6$; Figure 1A). Skin tonicity (resistance to traction, resistance to pinching and recovery after pinching) significantly improved by
$33 \%$ in comparison with the baseline value on the side treated with Mel creams only (from $3.0 \pm 0.5$ to $2.1 \pm 0.4$; Figure $1 \mathrm{~B}$ and C). Crow's feet photographic severity score was reduced significantly $(p=0.05)$ by $-15 \%$ with the creams in comparison with both baseline value $(2.3 \pm 0.2$ vs. $2.7 \pm 0.3)$ and value of the non-treated side after 3 months. Surface microrelief photographic score was significantly reduced by $-26.5 \%$ at the end of treatment in comparison with both baseline value (2.4 \pm 0.5 vs. $3.4 \pm 0.6 ; p=0.001$; Wilcoxon test) and value of the control side at month $3(2.4 \pm 0.5$ vs. $3.3 \pm 0.6 ; p=0.001$; Mann-Whitney $U$ test). Significant improvements were also observed starting from month 1 on the sides treated with the active product (ANOVA test; data not shown). For all the skin parameters evaluated, nonstatistical significant modifications have been observed in the control side comparing baseline and 3-month values. Figure 2 shows the evolution of the Spiderming graph with a significant reduction in the total area after active treatment in comparison with baseline and nontreated sides. Skin profilometry evaluation showed a $-13 \%$
A

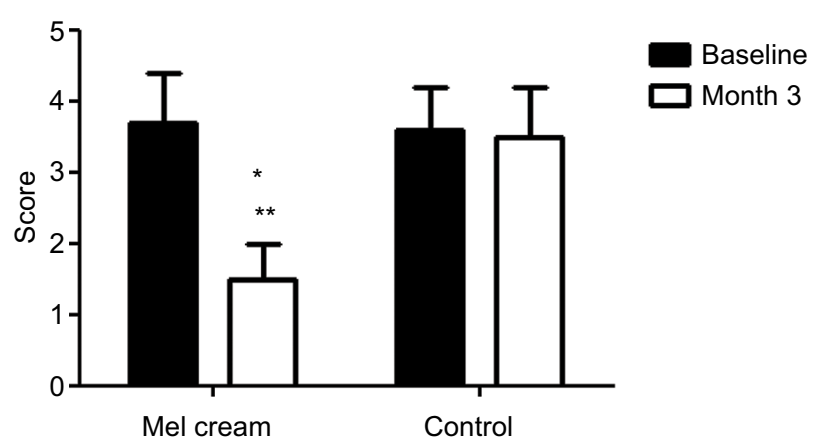

B

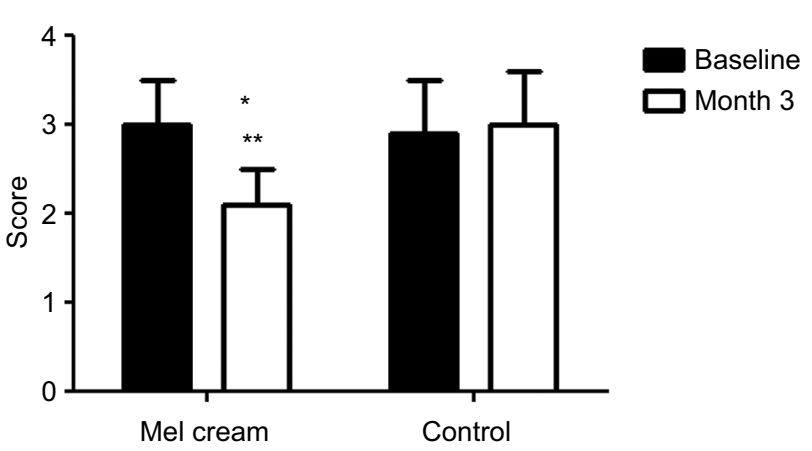

C

Skin tonicity: recovery after pinching

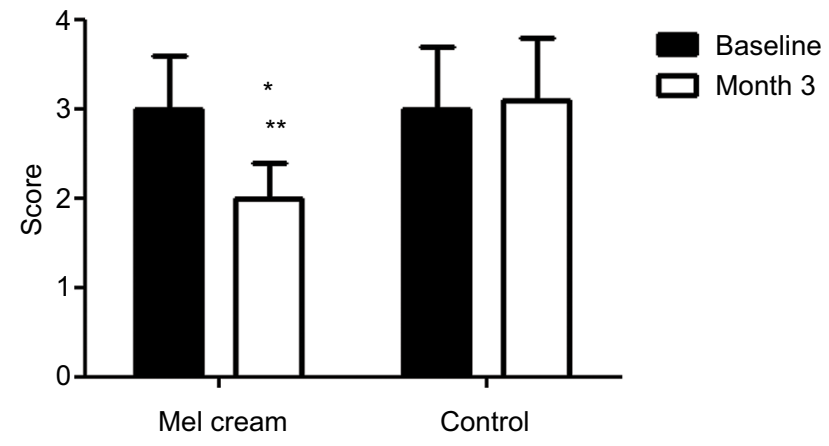

Figure I Evolution of skin parameters and treatment.

Notes: (A) Skin dryness evaluation (score from 0: very hydrated to 5 : very dry; ${ }^{*} p=0.00 \mathrm{I}$ in comparison with baseline, Wilcoxon test; ${ }^{*} p=0.0 \mathrm{I}$ in comparison with the control side; Mann-Whitney $U$ test). (B) Skin tonicity evaluation: resistance to traction (score from 0 : very relevant to 4 : very weak; ${ }^{*} p=0.05$ in comparison with baseline, Wilcoxon test; ${ }^{* *} p=0.05$ in comparison with the control side, Mann-Whitney $U$ test). (C) Skin tonicity evaluation: recovery after pinching (score from 0 : very relevant to 4 : very weak; ${ }^{*} p=0.05$ in comparison with baseline, Wilcoxon test; ${ }^{*} p=0.05$ in comparison with the control side, Mann-Whitney $U$ test).

Abbreviation: Mel, melatonin. 


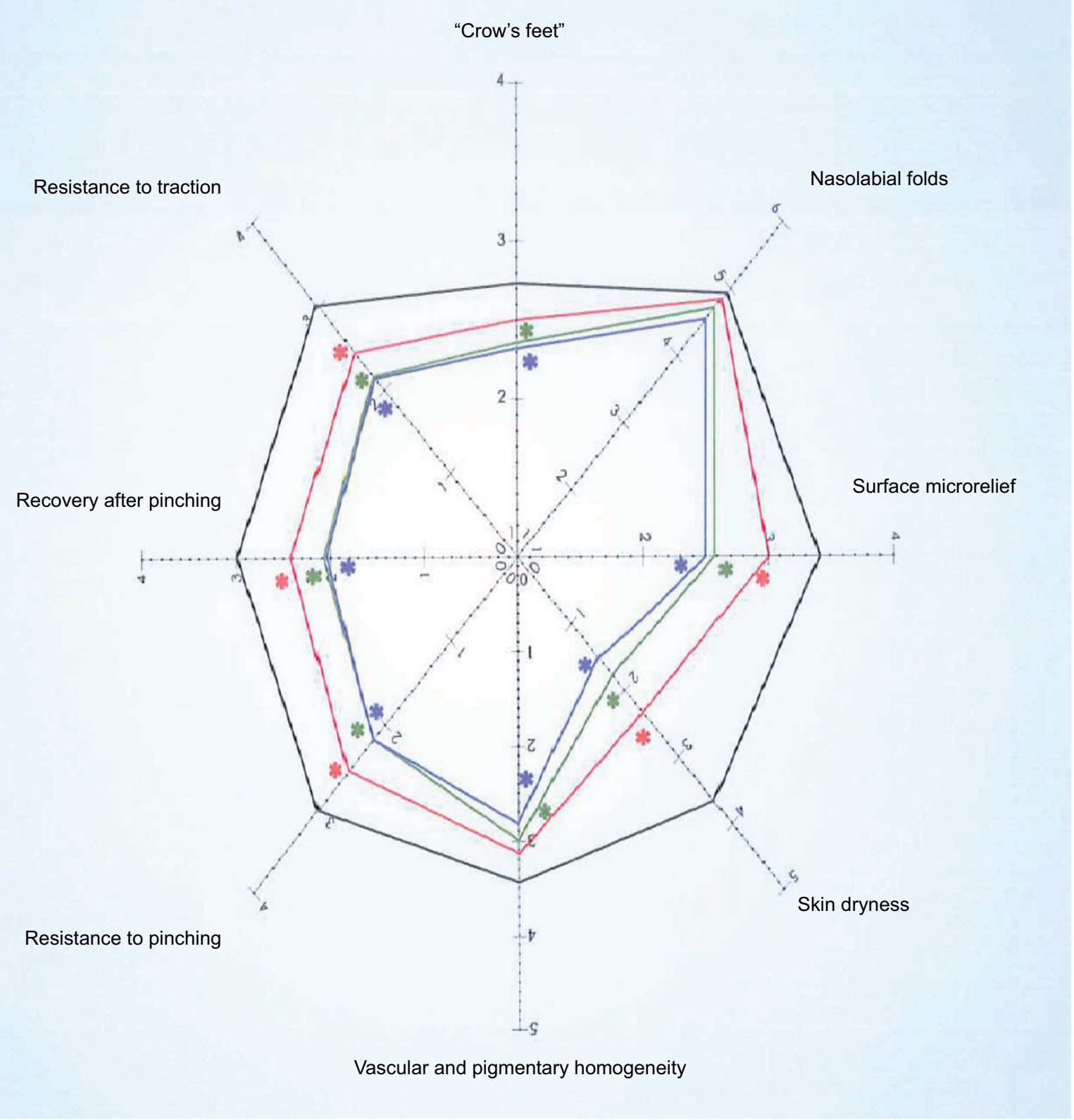

Figure 2 Evaluation of skin parameters.

Notes: The Spidermig ${ }^{\mathrm{TM}}$ graph. Black line: baseline values; red line: one month application of melatonin (Mel)-products; green line: two-month application of Mel products; blue line: three-month application of Mel-products.

reduction in roughness profile, $-13 \%$ reduction in wrinkles' maximum depth and $-7.2 \%$ reduction in total wrinkles' height in comparison with baseline and control sides. However, these reductions, although clinically relevant, did not reach a statistical significant difference. Figure 3 shows two subjects' pictures performed with Vectra at baseline and after 3 months of treatment. Both products (day and night creams) were well tolerated. No side effects were reported. Compliance to the treatment was $>90 \%$ in all the enrolled subjects.

\section{Discussion}

Mel is considered as a strong antioxidant molecule, and it is one of the most potent $\cdot \mathrm{OH}$ radical scavenger in nature. ${ }^{21}$ Experimental studies have shown that $\mathrm{Mel}$ is a stronger radical scavenger than vitamins $\mathrm{C}$ and E. ${ }^{22}$ Extrapineal Mel production has been demonstrated in bone marrow, cerebrospinal fluid, ovary, eye, lymphocytes, gastric mucosa and skin. ${ }^{23-25}$ Human skin and skin-derived cells can produce and metabolize Mel, and Mel exerts many effects on cell growth 

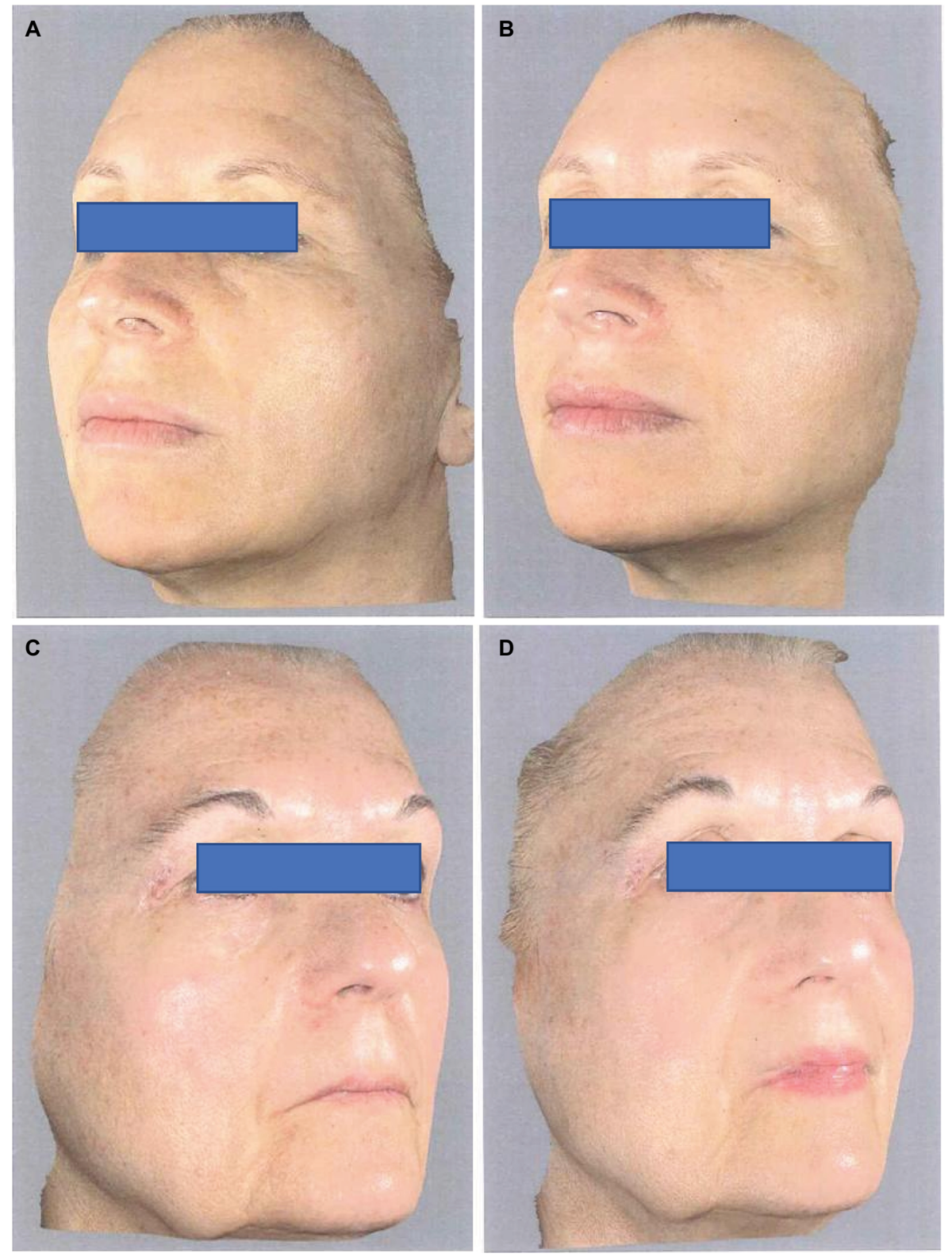

Figure 3 Vectra 3D images.

Notes: Subject I: (A) baseline and (B) after 3 months of active treatment (active products were applied on the left side). Subject 2: (C) baseline and (D) after 3 months of active treatment (active products were applied on the right side).

regulation and skin tissue homeostasis via Mel receptors MT1 and MT2. ${ }^{26}$ Skin can produce large amount of Mel. ${ }^{27,28}$ The protective effects of Mel against ultraviolet (UV) solar skin damage are mediated through direct and indirect radical scavenging and antioxidative enzyme-stimulating actions. ${ }^{29}$ Several data support the concept that at the skin level. Mel and its metabolites serve as protectors against physicochemical (oxidative damage, ultraviolet radiation [UVR], chemicals) and biological stressors. The antioxidant action of Mel has also genomic influences, regulating the expression of several genes. ${ }^{30}$ Mel stimulates both antioxidant enzyme activity and cellular mRNA levels for these enzymes. ${ }^{31}$ Mel could also protect DNA from oxidative damage..$^{32} \mathrm{Mel}$ can maintain mitochondrial membrane potential in UVR-exposed keratinocytes. ${ }^{33,34}$ Therefore, Mel is a good cytoprotective and antiaging molecule. ${ }^{35}$ As stated by Fischer et al, ${ }^{36}$ for application in clinical dermatology, exogenous Mel should be used topically rather than orally, since orally administered Mel appears in rather low levels in the blood due to prominent first-pass degradation in the liver, thus limiting skin access. Topical administration circumvents this problem. Therefore, in contrast with oral administration, topical application of Mel 
is considered meaningful, since it can penetrate the stratum corneum due to its distinct lipophilic chemical structure. In the present study, the tested products contain Mel molecules incorporated in lipospheres (Melatosphere) with the aim to further increase the skin penetration of the molecule. So far, no clinical data regarding the efficacy of topical Mel as an antiaging molecule has been reported. In our study, we evaluated for the first time, the efficacy of two Mel-based products in women with skin aging. The results we observed show that in comparison with the non-treated control side, these topical Mel formulations improve skin hydration and skin tonicity with a clinical improvement in the aspect of wrinkles. Some limitations should be taken into account in evaluating our results. First, this is a proof-of-concept study. Future trials with larger sample size are warranted to evaluate the real clinical effects of these products in the treatment of skin aging. A second limitation of this study is that this is not a double-blind trial. However, to increase the internal validity of our results, we adopted a split face, assessor-blinded study design.

\section{Conclusion}

In women with skin aging, Mel-based creams improved significantly skin tonicity and skin hydration with a significant reduction in skin roughness, supporting the skin antiaging effect of this molecule applied topically.

\section{Acknowledgment}

Difa Cooper Spa, IFC Group, supported this trial with an unrestricted grant.

\section{Author contributions}

AS conducted the trial performing visits and instrumental evaluations. MM was involved in study protocol design. Both authors contributed toward data analysis, drafting and critically revising the paper and agree to be accountable for all aspects of the work.

\section{Disclosure}

$\mathrm{MM}$ is an employee of Cantabria Lab, Difa Cooper. The other author reports no conflicts of interest in this work.

\section{References}

1. Slominski A, Baker J, Rosano TG, et al. Metabolism of serotonin to $\mathrm{N}$-acetylserotonin, melatonin, and 5-methoxytryptamine in hamster skin culture. J Biol Chem. 1996;271(21):12281-12286.

2. Slominski A, Wortsman J, Tobin DJ. The cutaneous serotoninergic melatoninergic system: securing a place under the sun. FASEB $J$. 2005;19(2):176-194.

3. Slominski RM, Reiter RJ, Schlabritz-Loutsevitch N, Ostrom RS, Slominski AT. Melatonin membrane receptors in peripheral tissues: distribution and functions. Mol Cell Endocrinol. 2012;351(2):152-166.
4. Slominski A, Chassalevris N, Mazurkiewicz J, Maurer M, Paus R. Murine skin as a target for melatonin bioregulation. Exp Dermatol. 1994;3(1):45-50.

5. Pandi-Perumal SR, Srinivasan V, Maestroni GJ, Cardinali DP, Poeggeler B, Hardeland R. Melatonin: nature's most versatile biological signal? FEBS J. 2006;273(13):2813-2838.

6. Tunali T, Sener G, Yarat A, Emekli N. Melatonin reduces oxidative damage to skin and normalizes blood coagulation in a rat model of thermal injury. Life Sci. 2005;76(11):1259-1265.

7. Slominski AT, Kleszczyński K, Semak I, et al. Local melatoninergic system as the protector of skin integrity. Int J Mol Sci. 2014;15(10): 17705-17732.

8. Bangha E, Elsner P, Kistler GS. Suppression of UV-induced erythema by topical treatment with melatonin (N-acetyl-5-methoxytryptamine). Influence of the application time point. Dermatology. 1997;195(3):248-252.

9. Kim TK, Kleszczynski K, Janjetovic Z, et al. Metabolism of melatonin and biological activity of intermediates of melatoninergic pathway in human skin cells. FASEB J. 2013;27(7):2742-2755.

10. Fischer T, Wigger-Alberti W, Elsner P. Melatonin in dermatology. Experimental and clinical aspects. Hautarzt. 1999;50(1):5-11.

11. Slominski AT, Semak I, Fischer TW, et al. Metabolism of melatonin in the skin: why is it important? Exp Dermatol. 2017;26(7):563-568.

12. Kleszczynski K, Fisher TW. Melatonin and human skin aging. Dermatoendocrinol. 2012;4(3):245-252.

13. Lane EA, Moss HB. Pharmacokinetics of melatonin in man: first pass hepatic metabolism. J Clin Endocrinol Metab. 1985;61(6):1214-1216.

14. DeMuro RL, Nafziger AN, Blask DE, Menhinick AM, Bertino JS Jr. The absolute bioavailability of oral melatonin. J Clin Pharmacol. 2000;40(7):781-784.

15. Fischer TW, GreifC, Fluhr JW, Wigger-Alberti W, Elsner P. Percutaneous penetration of topically applied melatonin in a cream and an alcoholic solution. Skin Pharmacol Physiol. 2004;17(4):190-194.

16. Domb AJ. Lipospheres for controlled delivery of substances. Microencapsulation: Methods and Industrial Applications. Abingdon, UK: CRC Press. 2005:297.

17. World Medical Association, et al. Declaration of Helsinki. Ethical Principles for Medical Research Involving Human Subjects. 2008. Available from: http://www.who.int/bulletin/archives/79\%284\%29373.pdf.

18. Glogau RG. Photoaging and aging skin. Basic Clin Dermatol. 2004;28:65-72.

19. Monheit GD, Gendler EC, Poff B, et al. Development and validation of a 6-point grading scale in patients undergoing correction of nasolabial folds with a collagen implant. Dermatol Surg. 2010;36(suppl 3):1809-1816.

20. Sparavigna A, Tenconi B, De Ponti I, Guglielmini G. Evaluation of the activity and tolerability of a cosmetic treatment for the periocular area on the aging face: controlled clinical and instrumental evaluation vs. placebo. Cosmetics. 2014;1(2):105-116.

21. Tan DX, Chen LD, Poeggeler B, Manchester LC, Reiter RJ. Melatonin: a potent, endogenous hydroxyl radical scavenger. Endocr $J$. 1993;1:57-60.

22. Fischer TW, Scholz G, Knoll B, Hipler UC, Elsner P. Melatonin suppresses reactive oxygen species in UV-irradiated leukocytes more than vitamin C and trolox. Skin Pharmacol Appl Skin Physiol. 2002;15(5):367-373.

23. Carrillo-Vico A, Calvo JR, Abreu P, et al. Evidence of melatonin synthesis by human lymphocytes and its physiological significance: possible role as intracrine, autocrine, and / or paracrine substance. FASEB J. 2004;18(3):537-539.

24. Tan DX, Manchester LC, Reiter RJ, et al. Identification of highly elevated levels of melatonin in bone marrow: its origin and significance. Biochim Biophys Acta. 1999;1472(1-2):206-214.

25. Fischer TW, Sweatman TW, Semak I, Sayre RM, Wortsman J, Slominski A. Constitutive and UV-induced metabolism of melatonin in keratinocytes and cell-free systems. FASEB J. 2006;20(9):1564-1566.

26. Slominski A, Pisarchik A, Semak I, et al. Serotoninergic and melatoninergic systems are fully expressed in human skin. FASEB J. 2002;16(8): 896-898. 
27. Kim TK, Lin Z, Tidwell WJ, Li W, Slominski AT. Melatonin and its metabolites accumulate in the human epidermis in vivo and inhibit proliferation and tyrosinase activity in epidermal melanocytes in vitro. Mol Cell Endocrinol. 2015;404:1-8.

28. Slominski A, Fischer TW, Zmijewski MA, et al. On the role of melatonin in skin physiology and pathology. Endocrine. 2005;27(2):137-148.

29. Fischer TW, Scholz G, Knoll B, Hipler UC, Elsner P. Melatonin reduces UV induced reactive oxygen species in a dose-dependent manner in IL-3-stimulated leukocytes. J Pineal Res. 2001;31(1):39-45.

30. Mayo JC, Sainz RM, Antoli I, Herrera F, Martin V, Rodriguez C. Melatonin regulation of antioxidant enzyme gene expression. Cell Mol Life Sci. 2002;59(10):1706-1713.

31. Rodriguez C, Mayo JC, Sainz RM, et al. Regulation of antioxidant enzymes: a significant role for melatonin. J Pineal Res. 2004;36(1) $1-9$.
32. Sliwinski T, Rozej W, Morawiec-Bajda A, Morawiec Z, Reiter R, Blasiak J. Protective action of melatonin against oxidative DNA damage - chemical inactivation versus base-excision repair. Mutat Res. 2007;634(1):220-227.

33. Slominski AT, Zmijewski MA, Semak I, et al. Melatonin, mitochondria, and the skin. Cell Mol Life Sci. 2017;74(21):3913-3925.

34. Fischer TW, Zmijewski MA, Wortsman J, Slominski A. Melatonin maintains mitochondrial membrane potential and attenuates activation of initiator (casp-9) and effector caspases (casp-3/casp-7) and PARP in UVR-exposed HaCaT keratinocytes. J Pineal Res. 2008;44(4):397-407.

35. Armstrong SM, Redman JR. Melatonin: a chronobiotic with anti-aging properties? Med Hypotheses. 1991;34(4):300-309.

36. Fischer TW, Slominski A, Zmijewski MA, Reiter RJ, Paus R. Melatonin as a major skin protectant: from free radical scavenging to DNA damage repair. Exp Dermatol. 2008;17(9):713-730.
Clinical, Cosmetic and Investigational Dermatology

\section{Publish your work in this journal}

Clinical, Cosmetic and Investigational Dermatology is an international, peer-reviewed, open access, online journal that focuses on the latest clinical and experimental research in all aspects of skin disease and cosmetic interventions. This journal is included on PubMed. The manuscript management system is completely online

\section{Dovepress}

and includes a very quick and fair peer-review system, which is all easy to use. Visit http://www.dovepress.com/testimonials.php to read real quotes from published authors

Submit your manuscript here: https://www.dovepress.com/clinical-cosmetic-and-investigational-dermatology-journal 